\title{
SIGNIFICATION ET PORTÉE DE L'IDÉE DU BIEN CHEZ PLATON
}

\author{
Begoña RAMÓN CÁMARA
}

Dédié au Professeur Francis Wolff, avec reconnaissance et affection

\section{Introduction}

Le but du présent travail est d'exposer succinctement une lecture de la conception platonicienne du Bien ( $\tau$ ò $\alpha \gamma \alpha \theta$ óv) qui permette de décrire la nature du Bien platonicien avec clarté et concision. Nous soutenons l'idée que le Bien présente une importante complexité sémantique qui, tout en prenant acte de la plurivocité, voire du caractère contradictoire de la notion, n'empêche pas toutefois de concilier les diverses significations et fonctions que le philosophe y façonne. Le présent travail tente donc d'apporter une définition du contenu et du sens de cette notion unitaire et cohérente du Bien.

Platon confère en effet à l'Idée du Bien une quadruple fonction éthique, politique, épistémologique et ontologique. En premier lieu, l'Idée du Bien est ce que « toute âme poursuit et dont elle fait la fin de tous ses actes» (Resp. 505d-e) et ce «qu'elle doit contempler à tout prix» $»(526 \mathrm{e})$ pour pouvoir donner un sens à sa propre vie ${ }^{1}$. Deuxièmement, le Bien est l'Idée que toute âme doit contempler pour savoir ce que c'est qu'un gouvernement politique juste et légitime, et pour conférer son sens à la vie humaine collective. En troisième lieu, l'Idée du Bien est aussi ce qui rend possible le savoir, dans la mesure où sa «lumière » rend le monde intelligible et l'homme intelligent. Enfin, le Bien est ce qui rend possible la vie de l'univers et sa continuelle conservation dans l'être. Nous citons le texte qui expose le plus brièvement la quadruple fonction causale attribuée au Bien :

En tout cas, c'est mon opinion, qu'aux dernières limites du monde intelligible est l'Idée du Bien, qu'on aperçoit avec peine, mais qu'on ne peut apercevoir sans conclure qu'elle est la cause universelle de tout ce qu'il y a de bien et de beau ; que dans le monde visible,

\footnotetext{
${ }^{1}$ La traduction est d'Émile Chambry (Platon, Euvres Complètes, t. VII, 1ère p.: La République, Paris, Les Belles Lettres, 1931).
} 
c'est elle qui a créé la lumière et le dispensateur de la lumière; et que dans le monde intelligible, c'est elle qui dispense et procure la vérité et l'intelligence, et qu'il faut la voir pour se conduire avec sagesse soit dans la vie privée, soit dans la vie publique ${ }^{2}$.

Néanmoins, bien que Platon répartisse l'Idée du Bien dans ces quatre idées ou conceptions distinctes, il est également vrai, aussi étrange que cela puisse paraître, qu'elles sont inséparables et convergent vers une seule idée générale. C'est ce qu'une leçon dialectique des Lois rappelle : le bon est une multiplicité, mais en même temps une unité. Ainsi, si on laisse échapper la manière dont il est et en quel sens il est - avertit Platon - on ne saisira jamais suffisamment son essence ${ }^{3}$.

Prenant bonne note de cette observation, on infère que, quant à la compréhension de ce sujet, on se doit d'une part de distinguer et d'expliquer avec clarté et précision chacune des fonctions spécifiques que le philosophe assigne au Bien. D'autre part, on doit se demander quel concept peut unir des choses à première vue si dissemblables et sans rapport entre elles comme le sont un premier principe du bonheur humain, dans son double et inséparable aspect individuel et collectif, un premier principe de la science et de la vérité, et un premier principe de l'être et de la constitution de l'univers. Car, dans une pensée unitaire comme celle de Platon, les dimensions éthique, politique, épistémologique et ontologique du Bien ne sont pas des choses distinctes et sans rapport entre elles, mais une seule et même activité montrant ses effets en plusieurs sphères de la réalité. La clé de cette unification peut être trouvée, comme le démontra R. L. Nettleship d'une manière magistrale, dans les critères propres à la vision téléologique de l'être, présente non seulement chez Platon, mais aussi dans la pensée grecque en général ${ }^{4}$.

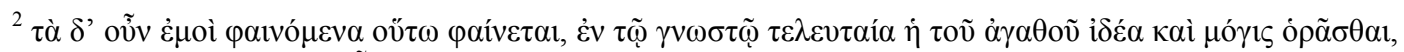

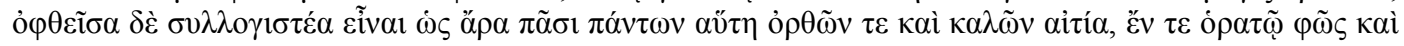

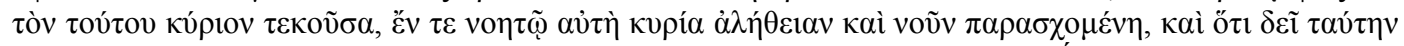

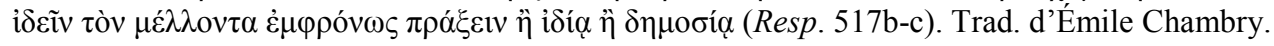

${ }^{3}$ Leg. 966a y $965 \mathrm{c}-\mathrm{e}$.

${ }^{4}$ Richard Lewis Nettleship, Lectures on the Republic of Plato, London, Macmillan, 1922, p. 218 sq.
} 


\section{Deux règles méthodologiques cruciales dans la théorie platonicienne du Bien}

Commençons par affirmer qu'il y a au moins deux constantes d'une importance vitale pour penser l'herméneutique du Bien. D'une part, la règle méthodologique selon laquelle ce sujet doit être abordé dans l'optique de la capacité de l'Idée du Bien à être

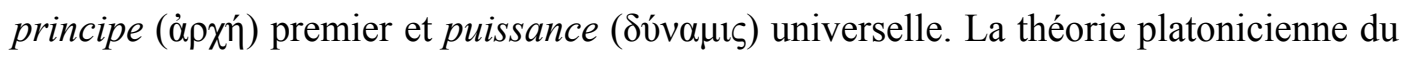
Bien est vouée à promouvoir, avant tout, la capacité principielle et causale de l'Idée du Bien au moyen de la description de son champ d'action et de ses effets. Ainsi instruit, le lecteur trouve un guide de lecture efficace de l'écriture platonicienne permettant de comprendre l'acception centrale de l'expression. D'autre part, cette autre règle méthodologique selon laquelle, pour aborder ce sujet lucidement, il faut savoir que, ni la condition maladive du langage ni la nature intrinsèquement fuyante de ce principe suprême ne permettent un exercice dialectique autre que celui d'entrevoir la puissance causale du Bien au moyen d'analogies sensibles. C'est une idée fausse - prévient le philosophe - que des yeux mortels puissent voir et connaître de manière satisfaisante un principe qui, quand on le regarde en face, produit la nuit en plein midi (Leg. 897d-e). Le pessimisme logique de Platon pousse la réflexion sur le Bien à se tourner vers la seule voie possible de l'allégorique. Mais, en même temps, la nécessité de recourir aux ombres du couchant finit par étendre puissamment les limites et les possibilités de la réflexion philosophique autour du Bien et par stimuler le raffinement littéraire. Ainsi, dans les moments dialectiques cruciaux des Dialogues, on voit défiler devant nos yeux plusieurs comparaisons qui décrivent éloquemment la féconde activité et les effets de la plus silencieuse des Idées avec une clarté magistrale et un désir de séduction qui, cependant, ne cachent pas un brin de doute et d'ironie d'un Platon dont le tempérament moral mène parfois à envelopper ses mots - écrits seulement pour des âmes qui ont des oreilles pour l'ineffable - d'un certain ton énigmatique.

\section{Sens du projet platonicien du Bien}

D'une part, l'essence du Bien - nous invite-il à penser - est ce qu'il fait. D'autre part, la tâche de la dialectique consiste à le montrer au lecteur en décrivant l'exubérante activité de l'Idée et ses produits. Le programme platonicien se propose comme but d'offrir une explication vraisemblable de l'origine et de l'intelligibilité de l'ordre 
général de l'univers à partir d'une seule Idée, à laquelle il confie une fonction épistémologique et ontologique mais aussi une fonction éthique et politique. Le philosophe fait appel à l'Idée du Bien alors qu'il est en quête d'une réponse à la question de la finalité de la vie tant individuelle que collective et d'une réponse à la question tant de la possibilité du savoir et de la vérité que du sens de l'origine et de la constitution de l'univers. Notre responsabilité en tant que lecteurs est alors de nous poser à notre tour la question de ce que le Bien doit être pour pouvoir réaliser la quadruple fonction soutenue par le philosophe ainsi que la manière concrète selon laquelle il doit être compris. Exposons donc la conception de chacune de ces fonctions et les clefs de la réponse de Platon à l'interrogation « qu'est-ce que le Bien ? ».

\section{Vision d'ensemble de l'unité et la multiplicité du Bien}

\subsection{Synopsis de la quadruple signification du Bien}

\subsubsection{Bref exposé de la signification éthique de l'Idée du Bien}

En premier lieu, le déploiement de la puissance éthique de l'Idée du Bien prend comme point de départ une conception de l'être humain comme être rationnel, c'est-àdire comme un animal qui se propose des fins et qui utilise des moyens, afin de découvrir quel est le vrai but de l'existence de l'homme.

Dans cet effort, Platon se voit assailli par le souci de démentir la thèse hédoniste - thèse à son avis terrible, honteuse et malheureuse - pour pouvoir ouvrir la voie à l'acceptation de la thèse opposée à propos de la nature du Bien. Entre le philosophe et le plaisir s'ouvre un abîme. Cette partie de la théorie est, dans un certain sens, une déclaration de guerre à la sensualité, en faveur de la paix que représente le fait de se consacrer à la vie neutre de l'intelligence délivrée de la douleur et du plaisir. Le vrai but de l'existence humaine, clame-t-il, ne peut pas être dans la simple expérience du plaisir sensuel - éternelle genèse insatiable et essentiellement rattachée à la douleur - mais plutôt dans la pratique des vertus cardinales, telles qu'elles sont définies dans la République, et dans la vie contemplative.

En s'appuyant sur les réflexions hippocratiques sur la santé, Platon soutient que la vertu de l'homme, comme la santé, est le produit d'un certain ordre et arrangement naturel, celui qui consiste en ce cas dans la coopération harmonieuse des différentes 
parties de l'âme qui a lieu quand chacune d'elles accomplit la fonction qui lui est propre. En outre, le parallélisme entre la santé et la vertu est utilisé à la manière

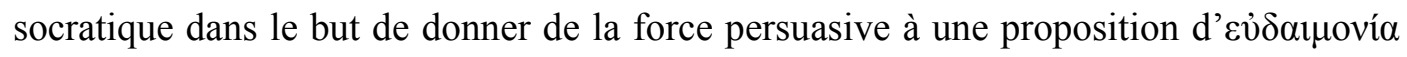
qui veut être un bien objectif pour l'ensemble de l'humanité. Si la vertu est « une certaine santé, beauté et bien-être de l'âme » (Resp. 444d-e), il semble judicieux, dit-il, de la préférer à l'injustice ou maladie de l'âme qui fait de la vie une espèce de mort au ralenti. Mais la vertu ainsi conçue est aussi le but naturel auquel répondent la naissance de l'homme et son passage sur la terre. Là réside le précieux enseignement - clef de l'humanisme platonicien - selon lequel la dignité de l'homme est dans la puissance d'une raison qui veut corriger et éduquer le plus possible notre irrationalité sans limites à l'aide indispensable d'un immense courage et de toute la force des bons sentiments.

Nous pensons aussi que dans cette philosophie l'idéal moral de maitrise de soi est une règle de vie génératrice d'unité intérieure et de stabilité. La première obligation d'un vrai homme - telle est l'exigence de Platon - est de devenir l'ami de soi-même. Et à cet effort de dureté et de probité envers soi-même - chacun est pour soi l'ennemi le plus proche et le plus terrible - doit se soumettre tout ce qu'il fait dans la vie, ou bien parce que ses habitudes, attitudes et actions contribuent à jouer « la symphonie de son âme » (591d), ou bien parce qu'elles évitent ce qui provoque son désaccord ou la menace. On prétend de cette manière forger une autosuffisance capable, d'un côté, de protéger le plus possible la liberté et la paix intérieures de l'individu face à son propre dérèglement et aux revers du sort; et d'un autre côté, capable de protéger le libre développement d'une vie consacrée à la recherche des Idées, la vie heureuse qui contribue à son tour à relativiser la nécessité des autres choses et des autres personnes. En échange, Platon promet le Bien - ici « le Bien » signifie le bonheur avec soi-même ou la jouissance d'un sentiment de quiétude et d'impassibilité de l'âme - malgré les malheurs que le destin nous réserve - le plus stable et continu possible pendant la totalité d'une vie. S'abandonner soi-même au destin sans opposer résistance - c'est, comme dit Socrate dans un passage merveilleux du Gorgias, «le généreux et le bon » (512d)- retenant dans l'esprit la seule vérité nécessaire: «qu'il n’y a pas de mal possible pour l'homme de bien $»^{5}$. 5 Apol. 41c-d. Trad. de Maurice Croiset (Platon, Euvres Complètes, t. I : Introduction, Hippias Mineur,
Alcibiade, Apologie de Socrate, Euthyphron, Criton, Paris, Les Belles Lettres, 1920). 


\subsubsection{Bref exposé de la signification politique de l'Idée du Bien}

En deuxième lieu, le déploiement de la puissance politique de l'Idée du Bien prend comme point de départ une explication de l'origine et de la constitution de la société afin de découvrir quel est le vrai but de l'existence de la société. Cette explication est basée sur deux faits naturels fondamentaux : le manque d'autarcie de l'individu humain pour produire ses moyens de vie et la sociabilité naturelle de l'homme.

En ce domaine, les pensées et les désirs de Platon sont destinés de manière explicite à condamner principalement la politique oligarchique dans les trois espèces militariste, libérale et absolutiste - qu'il distingue dans les livres VIII et IX de la République. Selon la thèse centrale de ces livres, cette conception et cette pratique de la vie publique sont trois caractéristiques essentielles des classes riches converties en catégorie politique ${ }^{6}$. Il s'agit de la sordidité, de la brutalité et de la rapacité imposées contre la multitude - le troupeau infâme - par une minorité - un troupeau de loups insatiables aux crocs ensanglantés avec la privatisation, la dette et la fiscalité. Ils le font au moyen des lois et du reste de l'appareil de l'État séquestré par eux et au moyen d'une politique extérieure basée également sur la domination ou sur l'assassinat commis pour piller et sur la « libre » circulation de l'or et de l'argent. Mais l'essence et le but de la plus grande des tâches ne sont absolument pas de s'emparer du bien d'autrui et de s'approprier des citoyens eux-mêmes. Cette finalité est de créer les conditions matérielles et culturelles qui rendent possible l'accomplissement du vrai but de la cité, à savoir, l'union entre les hommes en vue du Bien. Ici «le Bien» signifie le bonheur commun d'une collectivité qui a été unie politiquement à travers le partage des mêmes joies et des mêmes peines devant les mêmes vicissitudes.

En effet, la politique selon Platon est le savoir synoptique des fins à poursuivre par la cité afin d'avoir une vie en commun selon la vertu, pacifique et autosuffisante dans sa coopération unitaire pour satisfaire les besoins réels de ses membres et le reste de ses droits. Cette idée de la politique est liée à une conception de la culture comme

\footnotetext{
${ }^{6}$ Thèse en partie déjà exposée dans le Gorgias contre tout ce que la figure de Calliclès incarne et qui revient dans les Lois et dans quelques remarques de la Lettre VII.
} 
domestication des furies de l'intérêt privé et de la voracité ( $\pi \lambda \varepsilon 0 v \varepsilon \xi i ́ \alpha)$ grâce aux efforts du langage de la raison - « la sage persuasion » (Tim. 48a) - pour vaincre l'instinct et l'animalité. D'ailleurs, dans cette conception, l'idée d'ordonner et d'arranger la cité conformément au principe de fonction spécifique joue un rôle essentiel. Platon offre une double justification pragmatique et naturaliste de ce principe, bien que dans les Lois il l'abandonne dans son application particulière à la vie politique, afin de pouvoir démocratiser partiellement la vie publique. Depuis ces hauteurs, le philosophe lance ses foudres contre la «politique » oligarchique - contre un type de régime et de caractère qui jettent volontairement et subrepticement par terre, selon l'image de Platon, la raison et le courage - transformé de lion en singe (Resp. 590b) - aux pieds de l'avarice (553c-d). Parmi ses critiques les plus fréquentes, on trouve celle d'être une conception séditieuse, perverse et impie.

La principale conclusion à laquelle Platon parvient en ce domaine est que le seul moyen rationnel et efficace pour s'approcher de l'idéal régulateur du Bien dans son acception politique est la politique communiste, ou à défaut quelque chose de moins difficilement réalisable comme la politique socialiste, construite et gouvernée par un Conseil authentiquement aristocratique. Ce Conseil est composé par les meilleurs, par des philosophes qui, d'une part, n'ont de propriétés ni d'affaires commerciales et qui sont assujettis tout au long de leur vie à la plus stricte sobriété, et qui, d'autre part, font preuve du dévouement intellectuel le plus excellent, honnête et courageux. Il s'agit du seul mode de vie - à savoir, celui qui résume l'appel plein d'espoir de Platon au « retour à la vie au temps de Chronos »-qui peut couper ce que, dans sa promenade à travers l'histoire, le philosophe identifie comme la racine dernière de tout le problème de la politique et du devenir historique néfaste et affolé dans son ensemble. Ce problème est, d'après les dialogues politiques de Platon, la concentration du pouvoir économique, militaire et politique aux mains des classes dominantes par dépossession des classes dominées.

À cet égard, les écrits platoniciens apportent pour la première fois une bonne partie des valeurs et des principes directeurs qui deviendront constants dans la tradition intellectuelle communiste. L'œuvre platonicienne donne naissance aussi à un minutieux programme économique et éducatif adapté à ce type de valeurs et de principes qui inclut aussi bien des lignes stratégiques très générales ou pensées pour tous les temps que des mesures plus conjoncturelles. Mais il s'agit d'un programme pratique conçu, non seule- 
ment comme arme de défense et d'attaque contre le désordre oligarchique, mais aussi comme remède au désordre démocratique. Ceci est aussi décisif pour le sens de la tâche que se proposa Platon. Selon un profond et lucide diagnostic politique et psychologique de Platon, la démocratie est un type de régime également atteint d'un «mal morbide d'insatisfaction et insatiabilité » (Leg. 714a), mais un régime sans possibilités réelles de laisser libre cours à ce mal. Dans le cas de la dimension politique du Bien, Platon promet aux dialecticiens - « des chiens durs » (Resp. 422d) - la victoire finale, malgré son extrême difficulté et la mauvaise condition de la nature humaine, dans la guerre universelle pour le Bien. Il s'agit d'une guerre idéologique, oratoire et didactique universelle pour imposer, contre les hommes oligarchiques et leurs idées, la définition hégémonique du bonheur collectif. Platon est d'autre part convaincu qu'il n'existe pas d'autre salut pour le genre malheureux devant l'énormité du défi qu'il doit affronter.

\subsubsection{Bref exposé de la signification épistémologique de l'Idée du Bien}

En troisième lieu, le déploiement de la puissance épistémologique de l'Idée du Bien peut se résumer en deux idées fondamentales dans la théorie platonicienne de la connaissance, à savoir, la proposition d'un principe de l'ordre systématique total des Idées et la conception parallèle de la dialectique comme élévation à une vision du monde sub specie aeternitatis.

Dans sa quête de l'origine du savoir et de la vérité, ainsi que du rapport exact de dépendance que gardent l'intelligence et ce qui est appréhendé par elle avec leur cause originaire, Platon soutient l'idée que le Bien est la condition de possibilité du savoir en tant que principe producteur de la vérité de la chose connue et de la compréhension de l'intelligence. D'autre part, dans sa tâche de détermination du rapport de la philosophie avec les sciences, Platon établit l'idée que la dialectique est le seul savoir au sens fort du mot, c'est-à-dire un savoir universel, nécessaire et rationnellement fondé, dans la mesure où seule la dialectique s'élève jusqu'au «principe du tout » (511b). L'Idée du Bien est proposée à ce stade comme le principe dernier qui sert de fondement du savoir, même si Platon pense d'autre part que le savoir du principe suprême est hors de la portée de l'homme, ou qu'on peut tout au plus l'espérer sous la forme d'une étincelle, après avoir frotté péniblement les uns contre les autres éléments logiques et ontolo- 
giques de la connaissance (Ep. VII, 344b-c). Examinons cela de plus de près.

L'usage de l'expression «l'Idée du Bien » dégage dans ce contexte la croyance en une sorte de garantie de l'ordre rationnel du monde intelligible - un monde conçu comme un $\zeta \tilde{\omega}$ ov ou un tout doté d'une unité organique et complète - et de l'ordre du

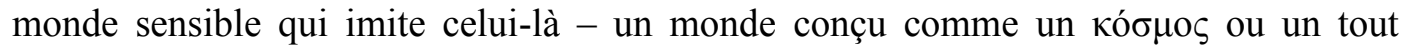
rationnel ordonné téléologiquement. Autrement dit, Platon semble penser que l'Idée du Bien est une sorte de puissance préservatrice de l'ordre et de l'arrangement rationnel entre les Formes qu'il faut présupposer pour parler de l'existence de la connaissance. Celle-ci est la première idée fondamentale du «moment» épistémologique de notre thème.

Il nous semble que c'est dans cette idée que réside la signification ultime de « la seconde navigation » annoncée dans le Phédon et appliquée dans le Timée. L'univers naturel ne peut être compris qu'en le contemplant à la lumière des Idées, porteuses d'une causalité formelle et finale, et, en dernière instance, de l'Idée du Bien, c'est-à-dire si l'étude de l'univers se focalise sur la compréhension de la fonction spécifique que tout être est appelé à réaliser. La dialectique est, dans ce sens, une méthode de recherche guidée par une conception de l'objet comme unité organique ou un ensemble de parties interconnectées pour la réalisation d'un $\tau \varepsilon ́ \lambda$ os et par la règle qui explique la fonction de chacune de ces parties et la manière dont elles convergent dans l'utilité principale de l'objet ou le bien du tout.

Or, la méthode dialectique est engagée dans une théorie de la causalité qui aspire à atteindre une vision panoramique du bien commun à toutes les choses, compte tenu du fait que la volonté de réunir et de penser dans son unité ce qui autrement est fragment, imperfection et obscurité doit forcément s'étendre à la totalité du réel. La fonction des êtres, l'activité propre qui les fait être ce qu'ils sont et dont la réalisation adéquate les rend bons, est déterminée par le lieu qu'ils occupent dans l'univers et ce lieu dépend luimême des rapports avec tout le reste. C'est pourquoi ce n'est qu'en parvenant à connaitre l'articulation du tout qu'on pourrait saisir pleinement l'activité de la partie. Dans cet autre sens, l'expression «l'Idée du Bien » serait - seconde idée fondamentale $\mathrm{du}$ « moment » épistémologique de notre thème - identique, d'une certaine manière, à l'idée d'un ensemble unifié de toutes les Idées et de leurs relations logiques, et la science dialectique à son tour serait identique à celle de l'idéal d'une vision synoptique de l'ordre rationnel qui gouverne l'univers et de la finalité de chaque chose. Cette 
universalité du savoir dialectique et sa capacité à tout réfuter permettent à Platon de le penser comme le seul savoir qui est réellement tel, motif qui lui permet de le placer « au plus haut, comme une espèce de couronnement du reste des enseignements » (Resp. 534e).

\subsubsection{Bref exposé de la signification ontologique de l'Idée du Bien}

En quatrième lieu, le déploiement de la puissance ontologique de l'Idée du Bien exprime la volonté de sonder l'origine et la raison dernière de l'ordre et la beauté de l'univers. La préoccupation dernière de cette investigation de la part de Platon, qui se dit, faisant la sourde oreille aux sophistes, qu'il n'est pas possible que l'univers soit un lieu aléatoire, arbitraire et étranger à l'intelligence humaine, est, comme nous le voyons dans le Timée et dans les Lois, celle de pouvoir invoquer l'objectivité du projet éthique et politique proposé en le fondant en dernière instance sur une explication ontologique qui persuade de l'essentielle rationalité de l'univers.

Il s'agit maintenant de prendre l'Idée du Bien en tant que cause dernière de l'être de tous les êtres. La clef pour comprendre cet aspect de l'Idée du Bien est donnée, une fois de plus, par le principe de fonction spécifique. On peut dire que Platon pose comme principe que l'Idée du Bien produit les autres Idées comme des paradigmes unitaires et parfaits, et donne aux choses, au moyen de leur action causale à la fois formelle et finale, l'ordre et la structure qui est la leur. Nous disions plus haut que les êtres se définissent par leur fonction spécifique et que la finalité qui leur est attribuée dépend du lieu qu'ils occupent dans le monde ; mais l'ordre et l'arrangement du monde dépendent à leur tour de l'Idée du Bien, la source et le principe causal dernier de cet ordre, auquel il est nécessaire - ainsi l'entend Platon - d'aboutir pour pouvoir démontrer que la nature est rationnelle et intelligible.

Nous pensons que derrière les diverses figures théologiques qui sont dans les Dialogues - le Bien du Phédon, l'Idée de la Beauté du Banquet, l'Idée du Bien de la République, l'Un du Parménide, l'artisan divin du Sophiste, la cause universelle du Philèbe, le démiurge du Timée et l'artisan du monde des Lois - est présente une même conception fortement unitaire qui peut être réduite à l'idée d'une Raison déterminée 
comme principe d'ordre et gouvernement de l'univers en vue de la réalisation du meilleur en termes universels.

Une caractéristique de cette conception ontologique est qu'elle projette sur toutes les choses le modèle d'explication de l'action humaine fondée sur la décision d'un sujet en vue du meilleur - conception défendue dans le Phédon. La volonté intelligente devient ainsi la cause en général. Conformément à cela, Platon demande qu'une véritable explication de l'univers doive accueillir la distinction entre causes finales et causes physiques : ces dernières, dépourvues par elles-mêmes de signification, sont les conditions dont se sert l'Intelligence pour réaliser ses desseins, tandis que ces desseins sont les seuls à rendre intelligibles les processus naturels.

En synthétisant à l'extrême la trame et le détail de la cosmogénèse que raconte le Timée, et qui est identique en essence au tableau métaphysique que peint le Philèbe, nous pouvons rappeler que la naissance de l'univers se représente comme l'heureux résultat de l'implantation de relations mathématiques dans le chaos indéfini et irrationnel par l'action d'une Intelligence décidée à obtenir l'autosuffisance, la plénitude et l'unité indissoluble de la nature. Si quelque chose peut définir l'éternel géomètre, c'est sans doute le goût qu'il montre pour introduire la proportion et la juste mesure là où elles font défaut.

Par ailleurs, précisons que Platon fait coïncider en cette notion de Raison une causalité finale doublée d'une causalité efficiente dernière de l'univers. Le Bien est, dans cet autre sens, l'objet d'une désirabilité universelle, ce qui revient à dire que tout être cherche à atteindre, à sa manière particulière et toujours selon les possibilités ouvertes par sa nature, la sereine satisfaction que seule son unité peut offrir. À cet égard, il convient de ne pas oublier non plus que, dans l'érotique philosophique de Platon, l'un des effets propres à l'Idée de la Beauté est celui d'inciter à la procréation notons que l'idée vient de la bouche d'un ascète - tant sensuelle que spirituelle.

Platon arrive à cette conception du Bien porté par un sentiment d'admiration devant la beauté du cosmos et par une méditation sur la régularité, le rythme et la finalité prédominants dans la nature. Ces faits induisent à soupçonner que « des calculs d'une si merveilleuse précision » (Leg. 967b) comme ceux auxquels répondent les êtres célestes et les processus naturels ne peuvent pas être dus à des puissances dépourvues 
d'intelligence $^{7}$. Mais le philosophe élabore aussi au moins deux argumentations avec lesquelles il veut renforcer la vraisemblance du postulat de l'existence et la souveraineté de la Raison. L'une est déployée dans le Philèbe et le Timée. Selon cette argumentation, l'intelligence immanente au cosmos ne peut être qu'une autoprojection de l'Intelligence transcendante au cosmos. L'autre est formulée dans les Lois contre la théorie présocratique et sophistique du caractère fortuit de l'univers. Cette autre argumentation s'occupe de glorifier la priorité de l'âme ou « le plus ancien et le plus puissant de tous les changements » (895b) par-dessus l'antiquité des éléments. À bien y regarder, le principe général dont dépend la théorie sophistique en question, à savoir le principe de l'antithèse selon laquelle s'excluent $\varphi v ́ \sigma ı \varsigma$ et vó $\mu \circ \varsigma$, se révèle comme une pensée fictive. La nature et l'art sont en quelque sorte la même chose, et tant l'une que l'autre sont le produit de la Raison, qui est le premier de tous les êtres, le premier tant à l'échelle temporelle qu'à l'échelle du bien, puisque l'exercice rationnel est la manifestation la plus haute, parfaite et précieuse de la nature. Vue selon l'optique des fonctions épistémologique et ontologique assignées au Bien, la philosophie de Platon est une vive exhortation à avoir confiance dans la rationalité de l'univers et dans le pouvoir apophantique de la dialectique.

Toute cette potentialité et dignité explicative de la dialectique du Bien fait que Platon confère à l'Idée du Bien un statut transcendantal unique. Nous croyons comprendre, en effet, que Platon lie expressément la transcendance de l'Idée du Bien à sa condition de premier principe et condition de possibilité de l'être et du savoir ainsi qu'à la nécessité méthodologique que l'inquiétude dialectique suscitée par la fondation de l'ontologie et de l'épistémologie puisse s'apaiser en atteignant un «fondement non fondé $)^{8}$.

À ce sujet, il nous semble que la République comme le Banquet s'opposent à une lecture néoplatonicienne qui obscurcirait complètement l'idée de la substantialité et de toute intelligibilité de l'Idée du Bien. Cela ne signifie pas, cependant, qu'on puisse mettre en doute la méfiance platonicienne à l'égard du langage, qui finit par mener le

\footnotetext{
${ }^{7}$ La traduction des mots entre guillemets est de Luc Brisson et Jean-François Pradeau (Platon, Les Lois, Livres VII à XII, Paris, Garnier Flammarion, 2006).

${ }^{8}$ J'emprunte à Francis Wolff l'expression entre guillemets (L'être, l'homme, le disciple. Figures philosophiques empruntées aux Anciens, Paris, PUF, 2000, p. 249).
} 
philosophe à une théologie négative. Cela ne signifie pas non plus qu'on puisse mettre en doute la reconnaissance d'un intense $\pi \alpha ́ \theta 0 \varsigma$ mystique partagé par le philosophe « divin » avec les néoplatoniciens. Ce $\pi \alpha ́ \theta$ os justement nous aide à comprendre que la thèse de la transcendance du Bien ne parle pas de l'éclipse de l'essence du Bien mais du fait que son essence ne peut pas s'égaler ni se rabaisser à l'un des sens dans lesquels le reste des choses participent de la réalité. Il s'agit d'un état d'esprit qui fait que le philosophe situe ce qu'il fête comme « le plus heureux de tous les êtres » (Resp. 526e) au-dessus de ce déplorable monde et qui le libère de toute souffrance et de toute attache avec le monde: le Bien ne doit connaitre qu'une sérénité et une impassibilité éternelles ${ }^{9}$. Car un sentiment de sublime indifférence est le propre d'un être inconditionnellement autarcique et parfait. Ce point des enseignements du Philèbe est, une fois de plus, une projection sur la figure du Bien d'une doctrine éthique cardinale apprise du plus merveilleux des hommes, Socrate.

Mais «la bonté » de la Raison ne se résout pas uniquement en son unité autosuffisante mais aussi en sa productivité désintéressée. Un mot, pour clore l'exposé de la fonction ontologique du Bien, à propos de la question du Timée et de la générosité du Bien comme raison dernière de l'existence de l'univers. Platon ne se contente pas d'assigner au Bien la liberté et l'indépendance de ce qui n'a besoin que de se connaître et de s'aimer soi-même; il souhaite en même temps l'impliquer dans l'existence d'un univers qui doit être bon ou rationnel - l'usage de l'adjectif «bon » ou « rationnel» exprime dans ce contexte, entre autres choses, le désir, de si longue portée métaphysique, de voir le monde comme quelque chose de "plein » de tout ce qui pouvait exister. Ainsi, quand il se demande pourquoi il existe un monde du devenir en plus du monde éternel des Formes, il trouve dans la bonté du démiurge, c'est-à-dire dans son être libre de malveillance contre aucun être, le dernier soutien de la pensée qui ne peut déjà être expliquée rationnellement. Cette absence d'envie ou de mesquinerie fait du Bien le «plus excellent de tous les êtres $»^{10}$. Mais Platon perçoit la contradiction à laquelle Aristote refusa de céder et tente de la surmonter en déduisant l'attribut de la générosité de l'attribut de l'autosuffisance. Celle-ci doit donner lieu à celle-là. Il s'agit

\footnotetext{
${ }^{9}$ La traduction de l'expression platonicienne est d'Émile Chambry (Platon, Euvres Complètes, t. VII, 1ère p., La République, trad. cit.).

${ }^{10}$ Resp. 532c. Trad. d'Émile Chambry.
} 
d'un point essentiel de la philosophie pratique de Platon qui réapparaît dans le Timée projeté sur le principe souverain dont la liberté absolue le situe au-dessus du monde.

\subsection{Synopsis de la notion unitaire et cohérente du Bien}

\subsubsection{Définition de l'Idée du Bien}

Il nous reste finalement à trouver une certaine unité à l'ampleur merveilleusement vaste et audacieuse d'une Idée du Bien puissamment représentée dans la méthode et le savoir dialectiques.

En effet, nous soutenons que derrière les quatre aspects présentés par l'Idée du Bien, il est possible d'entrevoir un seul et même principe produisant ses effets dans les différentes sphères de l'être. De même, nous soutenons que derrière les quatre dimensions de la théorie platonicienne du Bien, il y a une seule et même proposition philosophique, dont la défense constitue, nous semble-t-il, la raison d'être de la vie et de l'œuvre de Platon. Ce que nous avons fait au long de ces pages peut se résumer comme une tentative pour élucider la proposition, autant ontologique qu'axiologique, selon laquelle l'intelligence est et doit être souveraine. Nous pensons que le Bien est un seul et même principe dans tous les cas en partant de l'hypothèse qu'il est compris en dernière instance comme le principe unificateur de toute réalité, et que l'unité est comprise comme le but universel. Son sens varie selon le contexte : «le Bien » est l'idéal de la paix et du repos de l'âme dans l'éthique, ou l'idéal de la justice sociale et du Bien commun dans la politique, ou l'idéal d'un savoir total et systématique des Idées dans la théorie de la connaissance, ou l'idéal d'une Raison parfaite qui unifie ce qui est distinct en introduisant la proportion et la juste mesure en la matière dans l'ontologie. Mais dans tous les cas, le Bien est, en fin de compte, principe unificateur. Il est important de ne pas oublier que le fait de reconduire soigneusement la multiplicité à l'unité rationnelle est le but suprême pour cette forme de l'esprit. Nous atteignons ainsi le cœur de la philosophie platonicienne ou l'essence même de la dialectique. 


\subsubsection{Brève explication du rapport entre l'Idée du Bien et le reste des biens}

Nous croyons que les Dialogues montrent que le rapport entre l'Idée du Bien et les autres biens adopte la forme d'un rapport entre un premier principe causal, à la fois efficient et final, et ses effets.

À ce sujet, les textes adoptent un même ton destiné avant tout à montrer que l'excellence a beaucoup à voir avec la construction d'ordre et d'arrangement ou, dit en

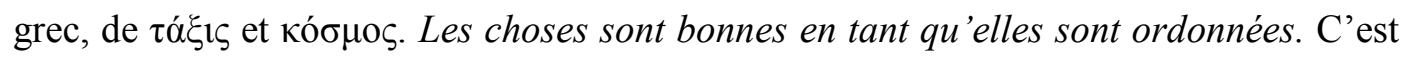
là la thèse générale pour laquelle plaide Platon, le lecteur devant entendre que le concept d'ordre est synonyme des concepts d'unité organique et de finalité. Platon nous invite ainsi à penser à l'idée d'une coopération harmonieuse entre les diverses parties ou fonctions des êtres en vue du développement plein de leurs capacités et de leurs fins propres. Du reste, nous pensons qu'une conclusion générale déductible de l'ontologie inspirée des artisanats et des mathématiques qu'élabore Platon est celle selon laquelle tout bien est une proportion arithmétique déterminée ou le produit de l'unification que la raison (soit la raison humaine soit la raison projetée sur le plus haut) réussit à créer et à préserver autant que possible entre les éléments différents de tel ou tel ensemble particulier.

Jusqu'ici nous n'avons traité que de la causalité efficiente de l'Idée du Bien. En ce qui concerne la causalité finale, il faut dire que l'Idée du Bien est également à la hauteur de l'engagement exigé par la dialectique platonicienne. En effet, l'Idée du Bien continue d'être une seule et même Idée dans les quatre fonctions éthique, politique, épistémologique et ontologique. Pensons au caractère de finalité universelle, autosuffisante et parfaite qui renferme le bonheur ou ce qu'on souhaite dans la vie pardessus tout. Mais aussi au fait qu'un dialogue désintéressé et sérieux sur le sens de notre passage sur la terre en tant qu'espèce ne peut que conclure résolument que rien n'est plus important que de réclamer la mise en œuvre du Bien commun et de la recherche de la perfectibilité de la nature humaine au moyen de la formation et la culture. Méditons maintenant sur l'appel de la théorie platonicienne de la connaissance à trouver de bonnes raisons pour ne pas voir dans l'ordre général de l'univers le résultat d'un accident, d'une absurdité ou d'un caprice, mais le résultat de ce qui devait être tel qu 'il est. Il faut arrêter à quelque principe la nécessité de remonter à des raisons ultérieures sur le pourquoi de l'ordre et de la constitution de l'univers. Les esprits platoniciens 
s'arrêtent au Bien. À ce Bien, notons-le finalement, dont on pense qu'il n'a ni pourquoi ni « en vue de quoi », puisque le parfait bonheur que représentent son autarcie et sa générosité sont la justification même de son existence. De cette manière nous pouvons conclure que, abordée sous tous les angles, l'Idée du Bien est toujours le point d'arrivée où les questions de la raison sur le pourquoi et le « en vue de quoi » touchent à leur fin. Dans ce sens, l'Idée du Bien est le destin dans lequel «l'inquiétude » intellectuelle et spirituelle du philosophe peut devenir enfin sérénité.

\subsubsection{Quelquesnotesutiles}

Nous pensons que seul ce type d'approche permet d'expliquer les fonctions du Bien et de s'appuyer sur l'ensemble des textes platoniciens. Pour une meilleure compréhension de ce type d'approche, il convient de rappeler les considérations suivantes.

En premier lieu, l'ordre ou l'unité auquel pense Platon vaut également pour n'importe lequel des trois domaines ou niveaux de la réflexion philosophique - homme, cité et univers. L'idée est qu'il y a un seul ordre qui gouverne l'univers selon lequel tout est pensé pour rendre possible l'exercice libre et «dominateur » de la raison sur ce qui est irrationnel. C'est uniquement grâce à l'existence de cet ordre que l'on peut parler de créer une rationalité dans la partie civile ou dans la partie personnelle. D'où la présence récurrente dans les Dialogues d'une optique pythagoricienne qui se refuse à détacher la réflexion à propos de ce qui est humain de la réflexion à propos de ce qui est cosmique. D'où aussi, en partie, le fait que Platon persévère dans l'idée qu'on peut penser la dialectique avec des garanties d'être une science unitaire, compte tenu du fait qu'elle a comme objet d'étude « défini »l'ordre universel.

En deuxième lieu, Platon prend comme point de départ l'idée selon laquelle l'ordre peut exister en vertu de la raison mais non en vertu du hasard. Aucune sorte d'unité ne peut prospérer, en vient-il à dire, si le soigneux et patient travail de la raison divine, cosmique et humaine n'est pas présent. Cela veut dire que la définition platonicienne de la notion de Bien passe par l'idée de quelque chose de vivant comme est la Raison, davantage que par l'idée de quelque chose d'immobile comme l'Unité. L’Idée du Bien est ce que Platon appelle « l'Intelligence qui a tout ordonné » (voṽ... 


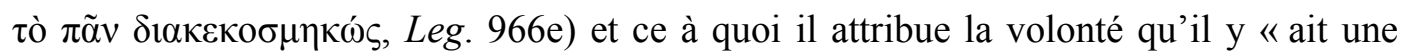
réalité heureuse dans la vie du tout $»^{11}$. La raison cosmique, quant à elle, est «une autoprojection » de cette figure transcendante. Pour sa part, la raison humaine est « une particule » plus ou moins puissante de la raison cosmique.

En troisième et dernier lieu, il importe de rappeler que Platon récolte ici en partie ce qu'Anaxagore, les pythagoriciens et Socrate ont semé au sujet de ce thème. Il nous semble que le Bien platonicien est en un certain sens le fruit logique de la synthèse

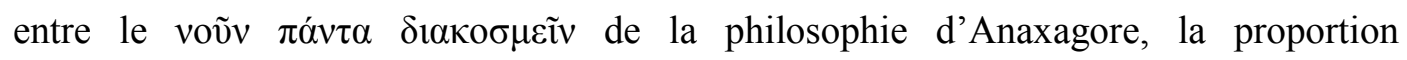
mathématique enseignée et convertie en essence de l'occupation du dieu par l'école pythagoricienne et l'attribution à la divinité par Platon de certaines propriétés de l'homme qui incarne l'essence de la sagesse, de certaines propriétés de la personnalité et de la pensée de Socrate.

\section{Bibliographie}

Platon, Euvres Complètes, t. I : Introduction, Hippias Mineur, Alcibiade, Apologie de Socrate, Euthyphron, Criton, traduction Maurice Croiset, Paris, Les Belles Lettres, 1920.

Platon, CEuvres Complètes, t. VII, 1ère p.: La République, traduction Émile Chambry, Paris, Les Belles Lettres, 1931.

Platon, Les Lois. Livres VII à XII, traduction Luc Brisson et Jean-François Pradeau, Paris, Garnier Flammarion, 2006.

Nettleship Richard Lewis, Lectures on the Republic of Plato, London, Macmillan, 1922.

Wolff Francis, L'Être, l'homme, le disciple. Figures philosophiques empruntées aux Anciens, Paris, Puf, 2000.

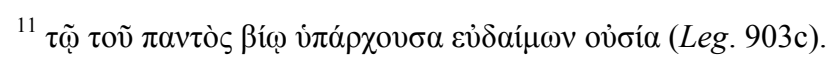

